\title{
Experimental Verification of the Impact of the 2nd Order Injected Signals on Doherty Amplifiers Nonlinear Distortion
}

\author{
$1^{\text {st }}$ Aleksandar Atanasković \\ Faculty of Electronic Engineering \\ University of Niš \\ Niš, Serbia \\ aleksandar.atanaskovic@elfak.ni.ac.rs \\ $3^{\text {rd }}$ Aleksandra Đorić \\ Faculty of Electronic Engineering \\ University of Niš \\ Niš, Serbia \\ aleksandra.djoric@elfak.ni.ac.rs
}

\author{
$2^{\text {nd }}$ Nataša Maleš Ilić \\ Faculty of Electronic Engineering \\ University of Niš \\ Niš, Serbia \\ natasa.males.ilic@elfak.ni.ac.rs
}

\author{
$4^{\text {th }}$ Djuradj Budimir \\ Wireless Communication Research Group, \\ University of Westminster, London, UK. \\ School of Electrical Eng., University of Belgrade \\ d.budimir@wmin.ac.uk; d.budimir@etf.rs
}

\begin{abstract}
In this paper, an asymmetrical Doherty amplifier fabricated in microstrip technology is tested in the experiments to verify the impact of the $2^{\text {nd }}$ order signal for the linearization prepared in baseband. The measurement set-up consists of three USRPs programmed by LabVIEW to generate the useful 64QAM signal and the signals for linearization that are set in amplitude and phase and modulate the $2^{\text {nd }}$ harmonic of fundamental carrier. The USRPs instruments should be synchronized for performing measurements for two scenarios: the signals for linearization are injected at input of the transistor within the main Doherty amplifier or are injected at its output.
\end{abstract}

Keywords-Doherty amplifier, baseband signal, second harmonic, linearization, experimental verification

\section{INTRODUCTION}

Doherty amplifier (DA) is very adequate topology for applications in modern wireless communication systems, especially in $5 \mathrm{G}$ due to its performances regarding the high efficiency and linearity.

In literature, the various solutions for the DA linearization can be found. In the contemporary technology, the most popular and applicable is digital predistortion technique [1], [2]. In order to linearize power amplifiers PA, single stage and DA, the authors of this paper have been processed the $I$ and $Q$ components of the useful signal, shaping them adequately in the $2^{\text {nd }}$ order nonlinear signals, whose amplitude and phase angles are set on the required values in the baseband. These signals then modulate the $2^{\text {nd }}$ harmonic of the fundamental carrier. The linearization signals formed in this manner are led at the gate and drain (input and output) of the amplifier transistor to decrease the nonlinearity of the power amplifier. The results of the linearization were predominantly analyzed in simulation software for the single stage PA [3], and the two-way DA [4] in terms of the input signal power and also in experiments. Measurements included in [4], which carried out on a DA, compare three cases - when the signals for linearization are inserted only at the input of the main DA amplifier transistor, only at its output and simultaneously at its input and output, but for the certain power levels.

In this paper, we represent the experimental results of the first and the second cases i.e. only input or output insertion of the linearization signals into the transistor of the main DA [5] for a range of 64QAM signal power. The measurements were performed to improve the adjacent channel power ration - ACPR at the range of dominant the third-order intermodulation products IM3, while we analyzed also behavior of ACPR parameter in the range of the fifth-order nonlinearities, IM5. In the future work, the experiments will be performed by using the simultaneous injection of the linearization signals at the input and output of the main Doherty amplifier transistor for the range of the input signal power.

\section{MEASUREMENT SET-UP}

In order to verify the linearization method developed within our research group, the measurement set-up shown in Fig. 1 was realized.

When forming the measurement system, it was considered that the measurement system should enable verification of the linearization methods, which generally uses two linearization signals, which after the digital processing in the baseband modulate the second harmonic of the fundamental signal. The measurement system was designed to provide possibility of generating three independent, synchronized signals - the fundamental signal and two linearization signals at the frequency of fundamental signal second harmonic.

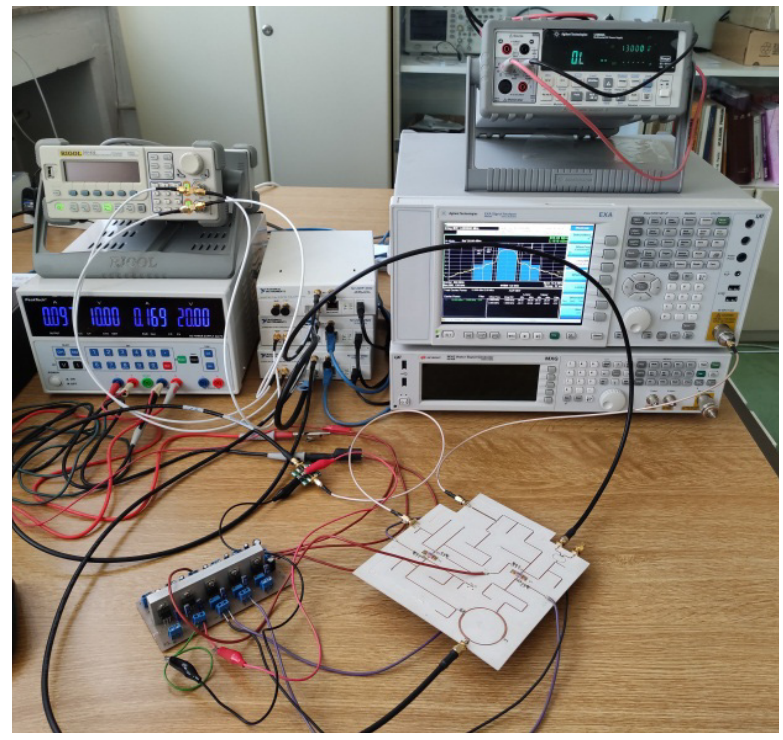

Fig. 1. Measurement set-up 


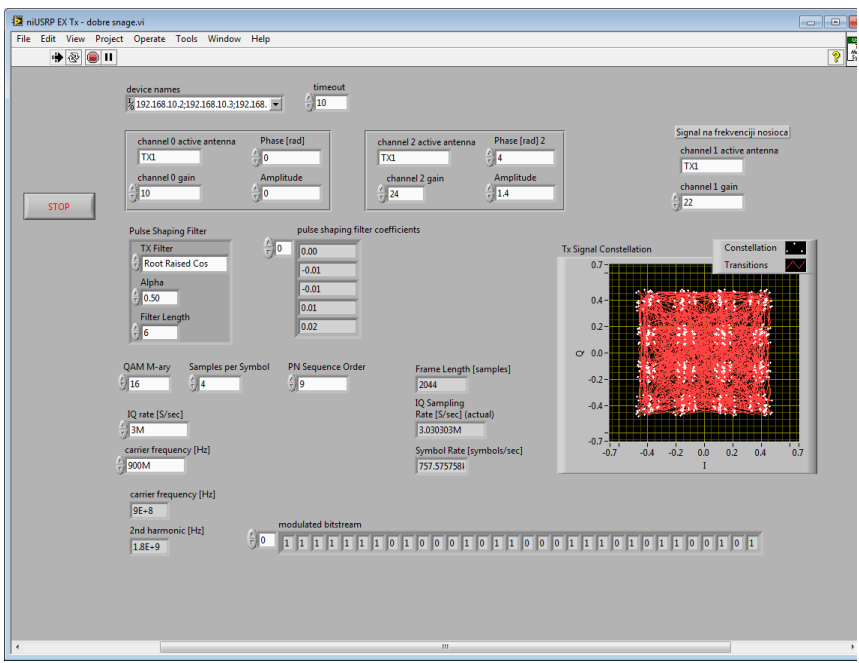

Fig. 2. Interface for management and control of the NI USRP devices.

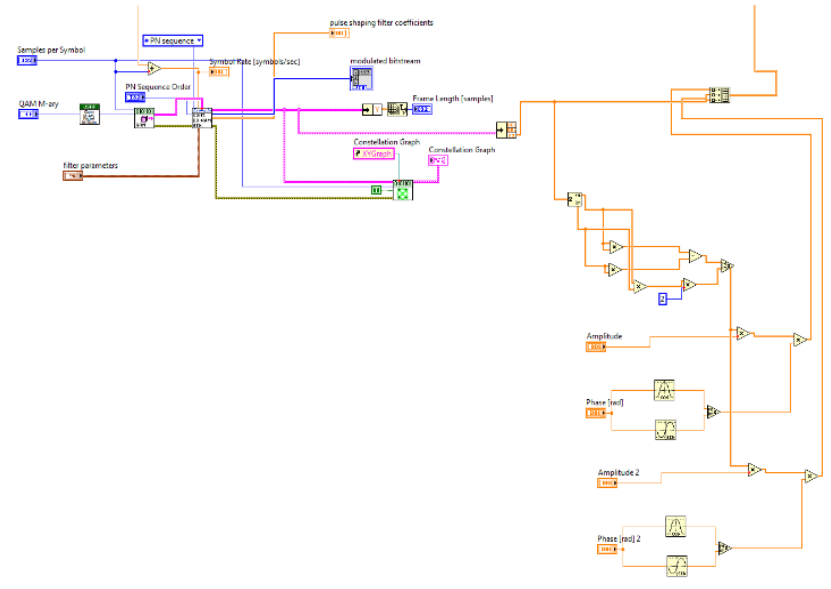

Fig. 3. Block diagram of implemented interface

Due to the low cost and good performances, softwaredefined radio (NI USRP) [6] in combination with the LabVIEW graphical programing environment [7] was chosen as the signal generation option. Two NI USRP 2920 models and one NI USRP 2922 model were used for the measurement system, which are connected to the computer via an Ethernet switch. The Keysight EXA Signal Analyzer N9010A was used for the signal measurement and analysis. The interface for management and control of the NI USRP devices was implemented in the LabVIEW environment (Fig. 2). The NI Modulation Toolkit, as a part of the LabVIEW, was used to generate the appropriate modulation signals. Using the designed interface, it is possible to set-up parameters related to the fundamental signal (operating frequency, modulation type - M-QAM $(\mathrm{M}=16,64,256)$, $I Q$ rate - signal bandwidth, samples per symbol, etc.) as well as the parameters related to the linearization signal amplitude and phase adjustment in the baseband. Also, for each of the generated signals, it is possible to independently adjust the gain of the NI USRP devices. Figure 3 shows block diagram details in the LabVIEW environment for the realization of the above mentioned functionalities.

In the experiments carried out in this paper when the signals for the linearization are injected either at the input or at the output of the main Doherty amplifier, only two NI USRP devices which are sinhronized by the use of MIMO

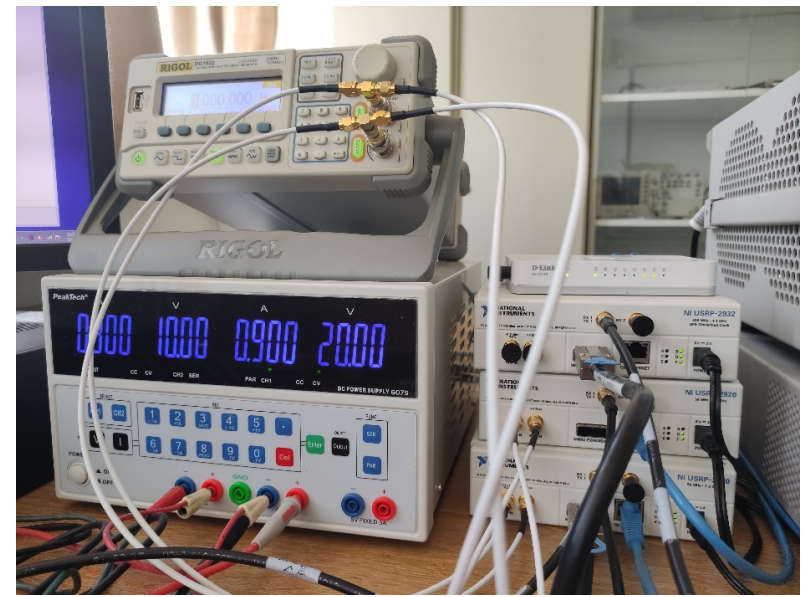

Fig. 4. Details of the NI USRPs synhronization.

cable may be used. Nevertheless, we set up the whole instrumentation required for the application of the linearization technique that implements both the input and output injection at the main DA transistor. Besides, this enabled us easier hardware application for the measurements done in this paper, avoiding exchange of the USPR, which generates and processes the signals for the linearization, between the input and output linearization approach.

A unique challenge during the implementation of the measurement system was the synchronization of the NI USRP devices. In accordance with the manufacturer's recommendations, the synchronization of the NI USRP devices was performed by a combination of MIMO cable (which was used to synchronize two of the three USRP devices - using MIMO EXPANSION input), while the third device was synchronized with the previous two via external referent $10 \mathrm{MHz}$ and $1 \mathrm{PPS}$ signals. A two-channel function/arbitrary waveform generator (RIGOL DG1022) was used as the generator of these signals, whereby the outputs from the function generator were distributed to the corresponding inputs of the NI USRP device (REF IN and PPS IN). Figure 4 shows the part of the measurement system where it can be seen how the NI USRP devices were synchronized, while Fig. 5 shows the LabVIEW block diagram which shows the details how the synchronization was implemented. When using the NI USRP with the LabVIEW environment, it is necessary to provide high processing power, large amount and high speed of memory as well as fast Ethernet connection to the computer on which the NI USRPs are connected, which is especially required if multiple USRP devices are used at the same time. The lack of any of these resources can significantly affect the reliable operation of the NI USRP and lead to frequent downtime.

\section{RESULTS}

The measurements of the linearization effects were performed on the Doherty amplifier fabricated in asymmetrical two-way topology (Fig. 6), which design and characteristics are given in [5]. The amplifier operates at $900 \mathrm{MHz}$ central frequency, and $9 \mathrm{~dB}$ of the maximal transducer gain was measured when the main amplifier is in class-AB and the auxiliary amplifier in class- $\mathrm{C}$ regime, characterized with $15 \mathrm{dBm}$ output power at 1-dB compression and $18 \mathrm{dBm}$ maximum output power. The amplifier characteristics are in the range of $\pm 0.5 \mathrm{~dB}$ across $10 \mathrm{MHz}$ bandwidth relating to their values at $900 \mathrm{MHz}$. 


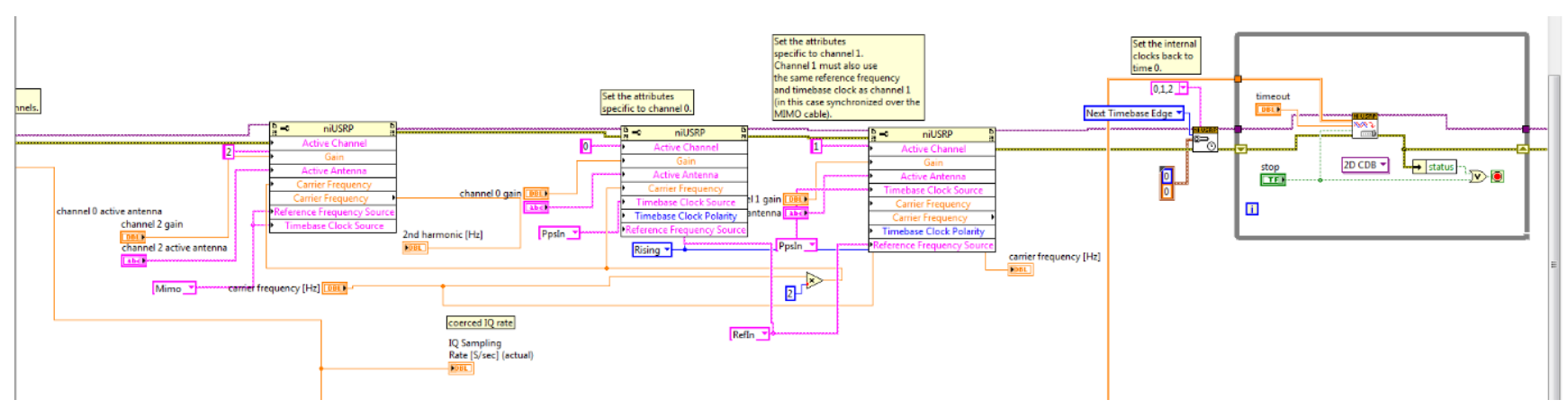

Fig. 5. Block diagram with synhronization details.

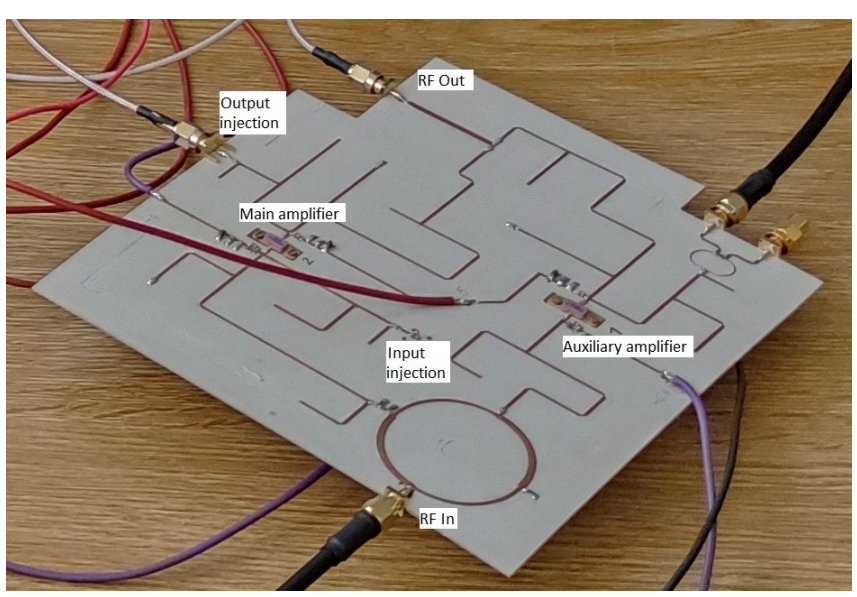

Fig. 6. Asymmetrical two-way Doherty amplifier

The Doherty amplifier was tested for the 64QAM signal with $2 \mathrm{MHz}$ channel bandwidth for diverse power levels of the output signal from $0 \mathrm{dBm}$ to $15 \mathrm{dBm}$.

The Figures 7 to 10 show the ACPRs measured before the linearization and when the linearization signals are put at the input of the main DA amplifier transistor (input injection/linearization) and when they are inserted at its output (output injection/linearization). The results of ACPRs are compared at $\pm 2 \mathrm{MHz}$ and $\pm 3 \mathrm{MHz}$ or adjacent channels (dominant IM3 products) and alternate channels (dominant IM5 products). We can notice that improvement of the ACPR in adjacent channels are better for the linearization of the DA by the output injection of the linearization signals especially for higher output power of the useful signal, i.e. for the input injection the maximum augmentation of the ACPR is approximately $5 \mathrm{~dB}$, whereas in the case of the output injection that is $7 \mathrm{~dB}$. It should be stressed that for the maximum considered output power, the output linearization approach achieves $5 \mathrm{~dB}$ the ACPR increase, when the input injection approach does not reach any results.

Observing the alternate channels, no evident ACPR improvement can be noticed for the output approach excepting at the output power levels close to maximum considered case where the ACPR becomes better by $5 \mathrm{~dB}$. The input injection linearization approach gives better results in the ACPR enhancement but maximally up to $2 \mathrm{~dB}$ in the lower output power levels of the useful signal, while at the maximum power does not approach any improvement.
It can be expected that the better linearization results of DA will be achieved when the injection of the signals for the linearization are applied at the input and output of the main amplifier transistor in Doherty configuration and that will be comparable with the DPD linearization results available in the literature.

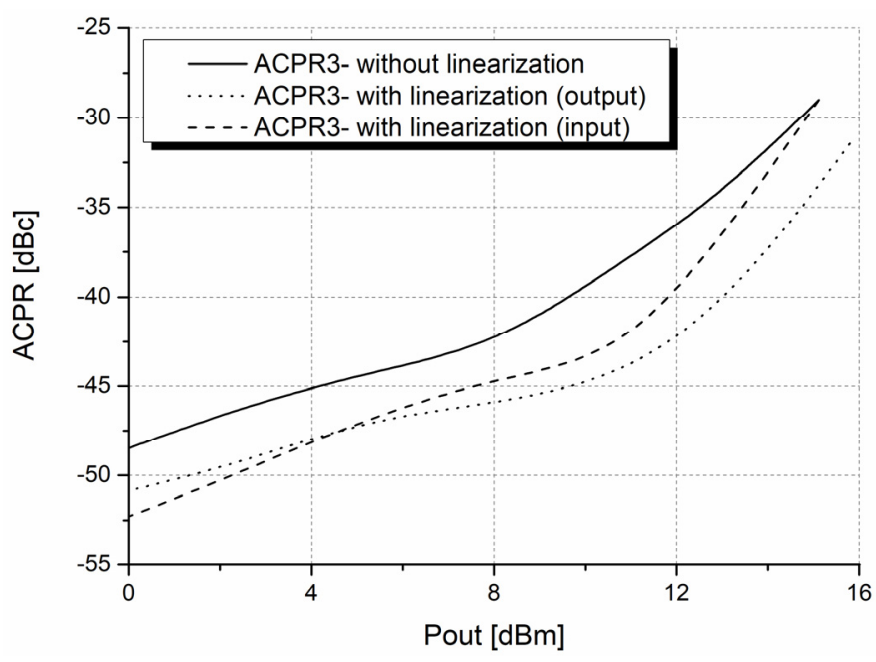

Fig. 7. ACPRs in terms of output fundamental signal power for 64QAM signal of $2 \mathrm{MHz}$ useful signal frequency bandwidth at - $2 \mathrm{MHz}$ offset from carrier over $1 \mathrm{MHz}$ frequency band.

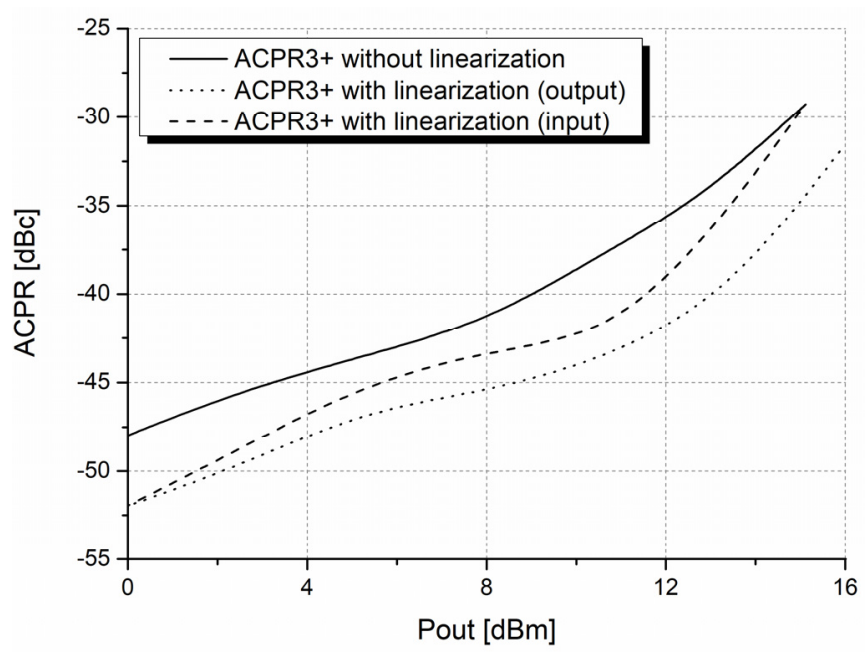

Fig. 8. ACPRs in terms of output fundamental signal power for 64QAM signal of $2 \mathrm{MHz}$ useful signal frequency bandwidth at $+2 \mathrm{MHz}$ offset from carrier over $1 \mathrm{MHz}$ frequency band. 


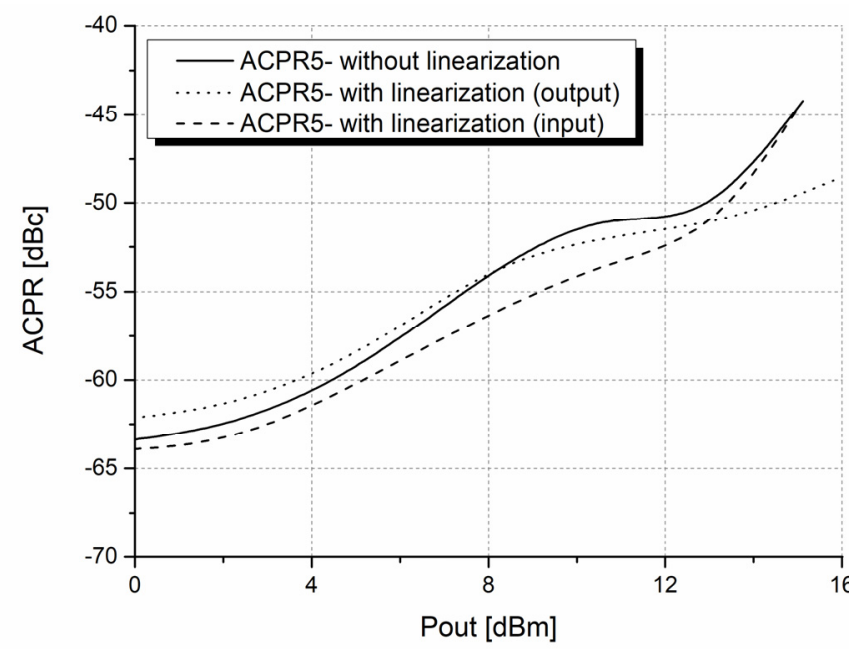

Fig. 9. ACPRs in terms of output fundamental signal power for 64QAM signal of $2 \mathrm{MHz}$ useful signal frequency bandwidth at $-3 \mathrm{MHz}$ offset from carrier over $1 \mathrm{MHz}$ frequency band.

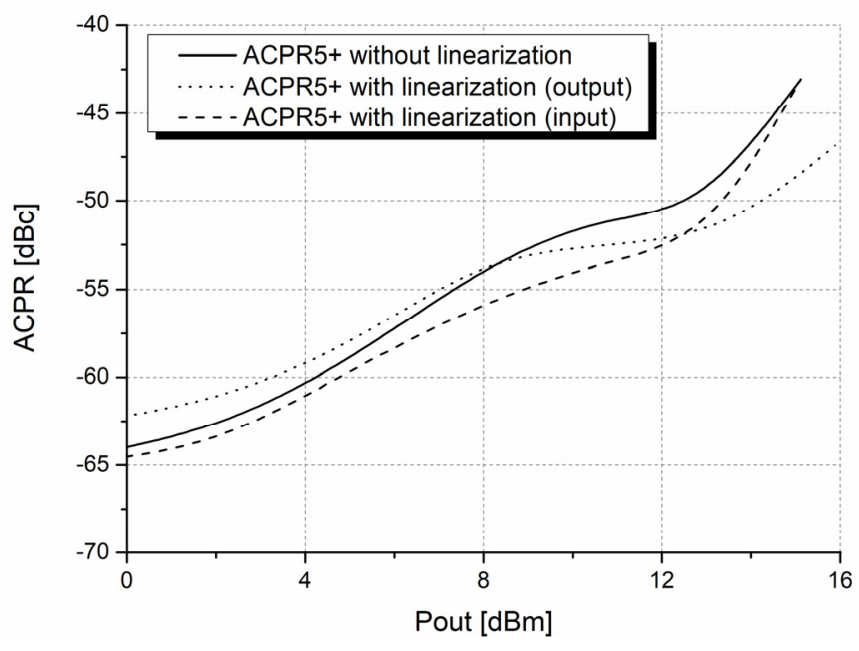

Fig. 10. ACPRs in terms of output fundamental signal power for 64QAM signal of $2 \mathrm{MHz}$ useful signal frequency bandwidth at $+3 \mathrm{MHz}$ offset from carrier over $1 \mathrm{MHz}$ frequency band.

\section{CONCLUSION}

The microstrip Doherty amplifier designed as two-way in asymmetrical topology is linearized by applying to approaches of the injection of the 2 nd order digital nonlinear signal adequately processed in the baseband, which then modulate the second harmonic of the fundamental carrier forming the linearization signals.

The useful 64QAM signals and signals for the linearization were generated and processed by the two NI USRP 2920 models and one NI USRP 2922 model programmed by the LabVIEW software. The Keysight EXA Signal Analyzer N9010A was used for the signal measurement and analysis. In the first experiments, the linearization was performed by the injection of the signal for linearization at the input of the DA main amplifier transistor, whereas in the second experiment those signals were led to the output of the transistor in the DA main amplifier. The parameter ACPR was measured at the adjacent $( \pm 2 \mathrm{MHz}$ ) and alternating channels $( \pm 3 \mathrm{MHz})$ over $1 \mathrm{MHz}$ frequency band for the 64QAM signal with the $2 \mathrm{MHz}$ channel bandwidth. On the basis of the measured results we can infer better influence of the output injection linearization considering adjacent channels. In the case of the alternate channels, slightly better improvement that is maximum $2 \mathrm{~dB}$ was measured in the input injection experiments. However, the output linearization attains significantly better results in the ACPR enhancement in the both adjacent and alternate channels for the higher output power of the useful signal close to saturation.

\section{ACKNOWLEDGMENT}

Fund for Science of Republic of Serbia supported this work, Grant: 6398983-Programme of collaboration of Serbian science with diaspora-project Digital Even-Order Linearization of 5G Power Amplifiers in Bands below 6GHz-DELFIN.

\section{REFERENCES}

[1] S. Jung, O. Hammi, and F. M. Ghannouchi, "Design optimization and DPD linearization of GaN-based unsymmetrical Doherty power amplifiers for 3G multicarrier applications", IEEE Trans. on Microwave Theory and Techniques, vol. 57, no. 9, pp. 2105-2013, September 2009.

[2] J.Xia, M. Yang, X. Zhu, "Linearized Asymmetrical GaN Doherty Power Amplifier with $100 \mathrm{MHz}$ instantaneous bandwidth at 3.5GHz", IEEE MTT-S International Microwave Workshop Series on RF and Wireless Technologies for Biomedical and Healthcare Applications, December 2013, Singapore.

[3] A. Atanasković, N. Males-Ilić, K. Blau, A. Đorić, and B. Milovanović, "RF PA linearization using modified baseband signal that modulates carrier second harmonic", Microwave Review, vol. 19, no. 2, pp. 119-124, December 2013.

[4] A. Đorić, A. Atanasković, N. Maleš-Ilić, and M. Živanović, "Linearization of RF PA by even-order nonlinear baseband signal processed in digital domain", International Journal of Electronics, Taylor \& Francis, vol.106, issue no.12, pp.1904-1918, December 2019.

[5] N. Maleš-Ilić, A. Atanasković, K. Blau, and M. Hein, "Linearization of asymmetrical Doherty amplifier by the even-order nonlinear signals", International Journal of Electronics, Taylor \& Francis, vol.103, issue no.8, pp.1318-1331, August 2016.

[6] Getting started guide USRP-2920/2921/2922, National Instruments, 2019, https://www.ni.com/pdf/manuals/376358b.pdf.

[7] Getting Started with LabVIEW, National Instruments, 2013, https://www.ni.com/pdf/manuals/373427j.pdf. 